[0212-7199 (2003) 20: 6; pp 301-303] ANALES DE MEDICINA INTERNA Copyright @ 2003 ARAN EDICIONES, S.L.

AN. MED. INTERNA (Madrid) Vol. 20, N. ${ }^{\circ}$ 6, pp. 301-303, 2003

\title{
Síndrome de vena cava superior y carcinoma insular de tiroides: el stent como alternativa terapéutica paliativa
}

\author{
M. LORENZO-SOLAR, J. LADO-ABEAL, J. CAMESELLE-TEIJEIRO', M. GARCÍA- \\ VÁZQUEZ², J. CABEZAS-CERRATO
}

Servicio de Endocrinología y Nutrición, 'Anatomía Patológica y ${ }^{2}$ Radiología. Complejo Hospital Clínico Universitario. CHUS. Santiago de Compostela

SUPERIOR VENA CAVA SYNDROME AND INSULAR THYROID CARCINOMA: THE STENT AS A PALLIATIVE THERAPEUTIC ALTERNATIVE

\section{RESUMEN}

El síndrome de vena cava superior (SVCS) es una complicación rara del cáncer de tiroides, que se produce como consecuencia de la invasión mediastínica por el tumor o por la invasión intravascular del mismo con trombosis. Creemos describir el primer caso de carcinoma insular de tiroides (variante tumoral indiferenciada del ca. folicular) con SVCS resuelto mediante la colocación de un stent venoso, que deviene así una alternativa eficaz y menos agresiva que la quirúrgica.

Se trata de un varón de 73 años que ingresa por un cuadro de disfonía y tumoración laterocervical derecha de dos meses de evolución; la PAAF de tiroides sugirió neoplasia folicular realizándose tiroidectomía total revelando el examen histológico un carcinoma insular de tiroides y administrándose una dosis ablativa de $100 \mathrm{mCi}$ de $\mathrm{I}^{131}$. Al año desarrolla un SVCS, observándose en la TAC recidiva tumoral, con elevación de las cifras de tiroglobulina ( $\mathrm{Tg}$ ) previamente normales. Descartada la indicación quirúrgica se administra radioterapia (52Gy) con despreciable respuesta clínica y agravamiento del cuadro a los pocos meses. Ante la imposibilidad de cirugía y radioterapia, se realizó por vía venosa braquial una flebografía torácico-mediastínica demostrándose obstrución venosa a nivel de la vena cava superior. Se realizó angioplastia y colocación de un stent desde vena innominada hasta vena cava superior; asistiéndose inmediatamente a una franca mejoría clínica. La flebografía de control a los tres meses mostró la permeabilidad del stent con flujo venoso rápido y desaparición de la circulación colateral cérvico-mediastínica.

PALABRAS CLAVE: Carcinoma Folicular de Tiroides. Síndrome de Vena Cava Superior. Stent.

\begin{abstract}
The superior vena cava syndrome (SVCS) is a uncommon complication of thyroid cancers. It is produced as consequence of the mediastinal spread of the tumor or by intravascularr invasion with thrombosis.

We describe a case of insular thyroid carcinoma with an SVCS solved by putting an intravenous stent.

The patient was a 73 year old male that consulted by aphonia and presence of a tumor in the right side of the neck of two months of evolution. The PAAF of thyroid suggested the diagnostic of "follicular tumor". A total thyroidectomy was performed on the patient and the sample histological study revealed the existence of a insular carcinoma. An ablative dosis of ${ }^{131} \mathrm{I}$ was adminestered to him. One year after the patient developed the SVCS. A TAC detected a tumoral relapses consistent with clinical syntoms, and was confirmed by a high level of Tg (with TgAntibodies -).

As the patient showed a light response to radiotherapy (52Gy), a thoracic flebografy was realized demostrating an extense uper cave venous obstruction. After having accomplished an angioplastia a long stent (20 mm wide) was putt into the uper cave vein that was followed by a fast clinical and radiological improvement. A new flebgraphy practiced three month later showed a rapid venous flux throuhgt the stent, and near totall disappereance of collateral circulation on thorax wall and mediastine
\end{abstract}

KEY WORDS: Follicular thyroid carcinoma. Thyroid insular carcinoma. SVC syndrome. Stent.

Lorenzo-Solar M, Lado-Abeal J, Cameselle-Teijeiro J, García-Vázquez. M, Cabezas-Cerrato J. Síndrome de vena cava superior y carcinoma insular de tiroides: el stent como alternativa terapéutica paliativa. An Med Interna (Madrid) 2003; 20: 301-303.

\section{INTRODUCCIÓN}

El síndrome de la vena cava superior (SVCS) es una rara complicación del cáncer de tiroides, produciéndose como consecuencia de la invasión mediastínica por el tumor con compresión de la vena cava o, más raramente aún, por la invasión intravascular y trombosis, siendo los tumores de extirpe folicular los que con mayor frecuencia desarrollan esta última complicación $(1,2)$.

Los carcinomas pobremente diferenciados constituyen un grupo de tumores situados morfológica y biológicamente en una posición intermedia entre los bien diferenciados (papilar y folicular) y el totalmente indiferenciado o anaplásico (3-8). Carcinoma insular es el nombre descriptivo propuesto por Carcangiu et al (3) en 1984, para una variante morfológica de los carcinomas pobremente diferenciados procedentes de las células foliculares, posteriormente descrito por otros autores $(6,7)$

En los pocos casos descritos en la bibliografía, la actitud terapéutica más empleada en la resolución del SVCS es la cirugía para la extracción del trombo y reconstrucción con injerto (10 ). Creemos describir el primer caso de carcinoma insular de tiroides (Medline,1972-2003) con síndrome de vena cava superior resuelto mediante la colocación de un stent 
intravenoso; procedimiento que pasa a constituir así una alternativa eficaz y menos agresiva que la quirúrgica.

\section{CASO APORTADO}

Varón de 73 años, sin antecedentes personales de interés, que en Agosto de 1994 ingresó en el hospital por un cuadro de disfonía y tumoración laterocervical derecha, dolorosa a la palpación y a la movilización del cuello, de dos meses de evolución. No existía historia de radiación previa de cuello. A la exploración física presentaba una tumoración situada a nivel del lóbulo tiroideo derecho de unos $6 \mathrm{~cm}$ de diámetro máximo, de consistencia dura, dolorosa y adherida a planos profundos. Se realizó PAAF tiroidea que fue sugestiva de neoplasia folicular de tiroides; se decide realizar tiroidectomía total con vaciamiento ganglionar radical derecho. La pieza resecada pesó $134 \mathrm{~g}$, y la sección mostró un tiroides distorsionado por múltiples nódulos grisáceos con focos de necrosis. El examen histológico reveló una neoplasia infiltrativa en la que destacaba la presencia de nidos bien definidos (insulae) (Fig. 1A), de configuración redondeada u oval, compuestos de una población monótona de pequeñas células con núcleos redondos y nucleolos pequeños. El carcinoma mostró una elevada actividad mitótica, importantes áreas de necrosis ubicadas sobre un patrón periteliomatoso (Fig. 1B), invasión de vasos de diferentes tamaños e infiltración de los tejidos blandos adyacentes a la glándula tiroides. Se hizo inmunohistoquímica usando la técnica de estreptavidin-biotin-peroxidasa (Dako, Glostrup, Denmark) fijando con formalina y disolviendo en parafina las muestras de tejido. El estudio inmunohistoquímico en las células neoplásicas fue positivo para queratinas de bajo peso molecular (Ab. monoclonal CAM 5.2, Becton-Dickinson, Mountain View, CA, prediluted), tiroglobulina (Ab. monoclonal, Dako, 1:20), proteina p53 (monoclonal, BioGenex, San Ramon, CA, 1:50) (Fig. 1A), y oncoproteina bcl-2 (Ab. monoclonal, Dako, 1:5). Fue en cambio negativo para calcitonina (Ab. policlonal, BioGenex, 1:2000), cromogranina (monoclonal, BioGenex, 1:10), y antígeno carcinoembrionario (Ab. monoclonal, Dako, 1:30). Se observó linfadenitis inespecífica sin metástasis tumoral en 17 ganglios linfáticos examinados.

Transcurridas cuatro semanas se realizó rastreo corporal con $6 \mathrm{mCi}$ de $I^{131}$ observándose dos restos captantes en la zona del lóbulo tiroideo izquierdo, procediéndose a la administración de una dosis ablativa. Se dio de alta al paciente con $200 \mathrm{mcg} /$ día de Levotiroxina y en buen estado. A los dos meses fué revisado en nuestra consulta, observándose que permanecía asintomático y con una tiroglobulina (Tg) de $20.69 \mathrm{ng} / \mathrm{ml}$ (n:0-60. Ab-AntiTg(-)). Un año más tarde demanda nuestra atención por un cuadro que describe como de hinchazón de cara, brazos, manos y
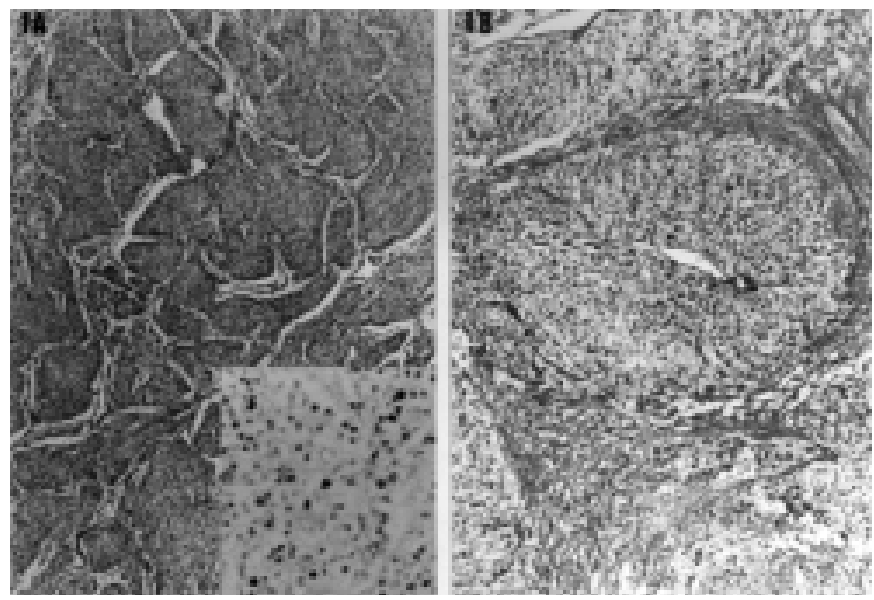

Fig. 1. En A se observan nidos celulares (insulae). En B se aprecia elevada actividad mitótica con varias áreas de necrosis ubicadas sobre un patrón periteliomatoso. región pectoral. La exploración física evidenció edema y enrojecimiento facial (12), del cuello y extremidades superiores, circulación colateral en la parte superior y anterior de hemitórax derecho, hombro y brazo derecho; zona inferior del tórax y superior del abdomen que se acentuaban con la elevación de los brazos por encima de la cabeza. La TC cervicotorácica reveló una masa mal delimitada en mediastino anterosuperior con obstrucción de los grandes troncos venosos cérvico-mediastínicos. La $\mathrm{Tg}$ en esta visita era de $114 \mathrm{ng} / \mathrm{ml}$ (Ab. antiTg (-). El Servicio de Cirugía Torácica contraindicó la intervención, por lo que se decidió realizar tratamiento con radioterapia (dosis total: $52 \mathrm{~Gy}$ ), con respuesta clínica nula.

En enero de 1996 era muy llamativo el SVC; la Tg era de 296 ng/ml (Ab. antiTg (-) y, la TC de cuello y mediastino mostró defectos de replección en la vena innominada derecha y vena cava superior (VCS), con extensa circulación colateral; se informó, además, de la presencia de pequeñas radioopacidades mediastínicas sugestivas de recidiva y/o metástasis ganglionares del cáncer folicular. Se realizó flebografía torácico-mediastínica vía venosa braquial bilateral, que demostró obstrucción venosa y circulación colateral cervical (Fig. 2A); las obstrucciones se localizaban principalmente en la vena áxilosubclavia derecha y vena cava superior que se extendía a la proximidad de la aurícula derecha. La circulación colateral drenaba hacia el territorio de la vena cava inferior. Se procedió, en primer lugar, a comprobar por vía femoral la accesibilidad de la vena innominada y VCS, y aunque existía cierta dificultad a nivel de la zona de obstrucción de esta última fue posible realizar angioplastia consiguiendo una dilatación de $18 \mathrm{~mm}$, con buen resultado angiográfico (Fig. 2B) y discreta mejoría clínica a las 24 horas. A las 72 horas fué implantado un stent (wallstent $20 \mathrm{~mm}$ x $11 \mathrm{~cm}$ ) que se extendía desde la vena innominada a la VCS hasta unos $1,5 \mathrm{~cm}$ de la aurícula derecha. Las maniobras fueron bien toleradas por el paciente, desapareciendo la sintomatología y disminuyendo los signos de obstrucción de la vena cava superior a los pocos días. Tres meses después se realiza flebografía de control en la que se observa la reexpansión y estabilidad de la prótesis (Fig. 2C), que permanecía permeable y con flujo rápido, habiéndose conseguido la desaparición casi completa de la circulación colateral cérvico-mediastínica (Fig. 2D).

\section{DISCUSIÓN}

El tumor aquí descrito reproduce un patrón histológico de carcinoma pobremente diferenciado (insular). Esta neoplasia parece ser más frecuente en unos países que en otros (5), ligera-

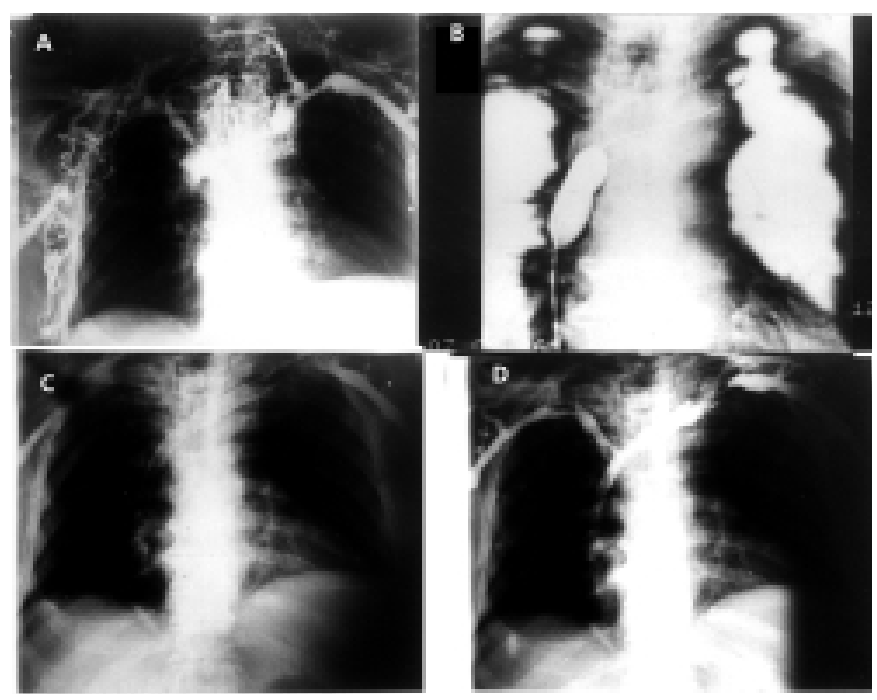

Fig. 2. Se muestran diferentes secuencias flebográficas antes de la inserción del stent $(A, B)$ y después de su inserción ( $C$ y $D)$. 
mente más frecuente en mujeres, y la edad media en el momento del diagnóstico es de 55 años (3). El carcinoma insular se caracteriza por un curso clínico altamente agresivo y a menudo letal con una supervivencia a los 5 años que varía entre el $45 \%$ y el $60 \%$ (3). Las zonas de metastización más frecuentes son los ganglios cervicales (64\%), ganglios mediastínicos (20\%), pulmón (44\%), y hueso (36\%) (3). La rápida y a menudo fatal evolución de esta variante requiere un tratamiento agresivo $(3,5)$. Al contrario que en el carcinoma indiferenciado, los tumores pobremente diferenciados generalmente presentan immunoreactividad para tiroglobulina y bcl-2, y concentran radioyodo (5), hechos que pueden ser utilizados para el diagnóstico y tratamiento. En relación a la proteína p53 nuestro hallazgo coincide con los de publicaciones previas $(8,9)$.

El SVCS en el contexto de un carcinoma folicular debido a la invasión de las grandes venas del cuello es una complicación poco frecuente (el primer caso fue descrito por Kaufman en 1879 (1), habiéndose publicado desde entonces solo 9 casos más (2), lo que unido a la escasa prevalencia del ca. insular, dificulta especialmente el establecimiento de cual ha de ser la actitud terapéutica más adecuada.
Es necesario recordar que la etiología del SVCS no siempre es tumoral (11), aunque es una complicación que aparece con cierta frecuencia en pacientes oncológicos, especialmente en tumores de origen pulmonar (13).

El procedimiento terapéutico tradicionalmente más empleado en los SVCS por procesos malignos es la radioterapia, no siempre eficaz (14) y con altas tasas de recidiva (lo que también sucedió en nuestro caso). La cirugía es una alternativa que en manos experimentadas puede resolver el problema $(15,16)$, pero no es siempre practicable, como en nuestro paciente.

La resolución de un SVCS secundario a malignidad mediante la colocación de un stent venoso es una alternativa eficaz sobre la que cada día se tiene más experiencia. En las series que han sido publicadas, se empleó este procedimiento sobre todo en el contexto de carcinomas bronquiales con extensión mediastínca; dentro de éstas series hemos encontrado algunos casos de SVCS por cáncer de tiroides, de los que no se especifica la variante histológica, con extensión medistínica que se resolvieron mediante stent (21-26).

\section{Bibliografía}

1. Graham A. Malignant epithelial tumors of thyroid with special reference to invasion of blood vessels. Surg Gynecol Obstet 1924; 39: 781-790.

2. Niederle B, Hausmanunger C, Kretschmer G, Polterauer P, Neuhold N, Mirza D et al. Intraarterial extension of thyroid cancer: tecnique and result of radical surgical aproach. Surgery 1990; 108: 951-6.

3. Carcangiu ML, Zampi G, Rosai J. Poorly differentiated ("insular") thyroid carcinoma. A reinterpretation of Langhans "wuchernde Struma". Am J Pathol 1984; 8: 655-668.

4. Rosai J, Carcangiu ML, Delellis RA. Tumors of the thyroid gland. Atlas of Tumor Pathology. 3rd series. Washington, Armed Forces Institute of Pathology, 1992: p. 123-133

5. Rosai J. Ackerman's Surgical Pathology. Vol 1. 8th edition. St Louis. MO: Mosby, 1996: p. 493-567.

6. Mizukami Y, Nonomura A, Michigishi T, Nogguchi M, Ohmura K, Nakamura S, Hoso M. Poorly differentiated ("insular") carcinoma of thyroid. Pathol Int 1995; 45: 663-8.

7. Nguyen GK, Akin MR. Cytopathology of insular carcinoma of the thyroid. Diagn Cytopathol 2001; 25: 325-30.

8. Soares P, Cameselle-Teijeiro J, Sobrinho-Simoes M. Immunohistochemical detection of p53 in differentiated, poorly differentiated and undifferentiated carcinomas of the thyroid. Histopathology 1994; 24: 205-210.

9. Furihata M, Ohtsuki Y, Matsumoto M, Sonobe H, Okada Y, Watanabe R. Immunohistochemical characterisation of a case of insular thyroid carcinoma. Pathology 2001; 33: 257-61.

10. Thompson NW, Brown J, Orringer M, Sisson J, Nishiyama R. Follicular carcinoma of the thyroid with massive angioinvasion: Extension of tumor thrombus to the heart. Surgery 1978; 83: 451-457.

11. Musumeci ML, De Pasquale R, Tedeschi A. Non malignant superior vena cava síndrome. Int J Dermatol 2000; 39: 934-6.

12. Burgdorff T, Douwes KE, Bogenrieder T, Szeimies RM, Hohenleutner U, Landthaler M, Stolz W. Superior vena cava syndrome: an important differential diagnosis in patients with facial edema. Acta Derm Venerreol 2001; 81: 205-6.

13. Gupta R, Gupta S. Oncologic emergencies: superior vena cava síndrome. Cleve Clin J Med 2002; 69: 744.

14. Wilford MR, Chertow BS, Lepanto PB, Leidy JW. Dramatic response

of follicular thyroid carcinoma with superior vena cava syndrome and tracheal obstruction to external-bean radiotherapy. Am J Med 1991; 90: 753-757.

15. Perez and Brown. Follicular carcinoma of the thyroid appearing as an intraluminal superior vena cava tumor. Arch Surg 1984; 119: 323-326.

16. Bordigoni L, Blin D, Magnan PE, Giudicelli R,Fuentes P. Ectatic tumoral thrombosis of the superior vena cava revealing thyroid cancer. Ann. Radiol 1992; 35: 559-563.

17. Rowell NP, Gleeson FV. Steroid, radiotherapy, chemotherapy ands stents for superior vena cava obstruction in carcinoma of the bronchus: a systematic review. Clin Oncol (R Coll Radiol) 2002; 14: 338-51.

18. Calderón MC, Lozano VM, Jaquez A, Villasenor C. Surgical repair of superior vena cava syndrome. Ann Thorac Surg 2001; 71: 1351-3.

19. Porte H, Metois D, Finzi L, Lebuffe G, Guidat A, Conti M, Wurtz A Superior vena cava syndrome of malignant origin. Which surgical procedure for which diagnosis? Eur J Cardiothorac Surg 2000; 17: 384-8.

20. Roberts JR, Bueno R, Sugarbaker DJ. Multimodality treatment of malignant superior vena caval syndrome. Chest 1999; 116: 835-7.

21. Kazushi k, Sonomura T, Mitsuzane K, Nishida N, Yang R, Sato M et al. Self expandable metalic stent therapy for superior vena cava syndrome : Clinical observations. Radiology 1993; 189: 531-535.

22. Shigeru F, Sawada S, Kuramoto K, Inoue $\mathrm{Y}$, Irie $\mathrm{T}$, Makita $\mathrm{K}$ el al. Gianturco stent placement in malignant caval obstruction: Analysis of factors for predicting the outcome. Radiology 1995; 195: 147-152.

23. Hennequin L, Fade O, Fays J, Bic JF, Jaafar S, Bertal A, et al. Superior vena cava stent placement: Results with the Wallstent endoprosthesis. Radiology 1995; 196: 353-361.

24. Lanciego C, Chacón JL, Julián A, Andrade J, López L, Martínez B Cruz M, García-García L. Stenting as first option for endovascular treatment of malignant superior vena cava syndrome. AJR Am J Roentgenol 2001; 177: 585-93.

25. Wilson E, Lyn E, Lynn A, Khan S. Radiological stenting provides effective palliation in malignant central venous obstruction. Clin Oncol (R Coll Radiol) 2002; 14: 228-32.

26. Rochais JP, Icard P. Stent placement in superior vena cava syndrome. Ann Thorac Surg 2003; 75: 158-61. 